\title{
Effects of Cardiovascular Risk Factor Variability on Health Outcomes
}

\author{
Seung-Hwan Lee ${ }^{1, *}$, Mee Kyoung Kim²,*, Eun-Jung Rhee ${ }^{3}$ \\ ${ }^{1}$ Division of Endocrinology and Metabolism, Department of Internal Medicine, Seoul St. Mary's Hospital, College of Medicine, \\ The Catholic University of Korea; ${ }^{2}$ Division of Endocrinology and Metabolism, Department of Internal Medicine, Yeouido St. \\ Mary's Hospital, College of Medicine, The Catholic University of Korea; ${ }^{3}$ Department of Endocrinology and Metabolism, \\ Kangbuk Samsung Hospital, Sungkyunkwan University School of Medicine, Seoul, Korea
}

Innumerable studies have suggested "the lower, the better" for cardiovascular risk factors, such as body weight, lipid profile, blood pressure, and blood glucose, in terms of health outcomes. However, excessively low levels of these parameters cause health problems, as seen in cachexia, hypoglycemia, and hypotension. Body weight fluctuation is related to mortality, diabetes, obesity, cardiovascular disease, and cancer, although contradictory findings have been reported. High lipid variability is associated with increased mortality and elevated risks of cardiovascular disease, diabetes, end-stage renal disease, and dementia. High blood pressure variability is associated with increased mortality, myocardial infarction, hospitalization, and dementia, which may be caused by hypotension. Furthermore, high glucose variability, which can be measured by continuous glucose monitoring systems or self-monitoring of blood glucose levels, is associated with increased mortality, microvascular and macrovascular complications of diabetes, and hypoglycemic events, leading to hospitalization. Variability in metabolic parameters could be affected by medications, such as statins, antihypertensives, and hypoglycemic agents, and changes in lifestyle patterns. However, other mechanisms modify the relationships between biological variability and various health outcomes. In this study, we review recent evidence regarding the role of variability in metabolic parameters and discuss the clinical implications of these findings.

Keywords: Variability; Glucose; Blood pressure; Body weight; Lipids; Mortality; Cardiovascular diseases

\section{INTRODUCTION}

There are innumerable studies supporting the principle of "the lower, the better" for cardiovascular (CV) risk factors in terms of outcomes. Multiple cholesterol management sources have suggested lowering low-density lipoprotein cholesterol (LDLC) levels to the greatest extent possible to prevent cardiovascular disease (CVD) in high-risk groups in accordance with recent

Received: 10 May 2020, Revised: 7 June 2020, Accepted: 10 June 2020

Corresponding author: Eun-Jung Rhee

Department of Endocrinology and Metabolism, Kangbuk Samsung Hospital, Sungkyunkwan University School of Medicine, 29 Saemunan-ro, Jongno-gu, Seoul 03181, Korea

Tel: +82-2-2001-2485, Fax: +82-2-2001-1588, E-mail: hongsiri@hanmail.net
CV outcome trials that showed additional benefits for CVD prevention from using proprotein convertase subtilisin/kexin type 9 (PCSK9) inhibitors to lower mean LDL-C levels to $30 \mathrm{mg} / \mathrm{dL}$ [1-3]. In addition, debate continues regarding lower blood pressure (BP) control targets based on the Systolic Blood Pressure Intervention Trial (SPRINT) results [4,5]. In patients with diabetes, although concerns remain regarding the deleterious effects of hypoglycemia on CV outcomes and cognitive dysfunc-

\section{Copyright $\odot 2020$ Korean Endocrine Society}

This is an Open Access article distributed under the terms of the Creative Commons Attribution Non-Commercial License (https://creativecommons.org/ licenses/by-nc/4.0/) which permits unrestricted non-commercial use, distribution, and reproduction in any medium, provided the original work is properly cited. 
tion, strict glycemic control with a glycated hemoglobin (HbAlc) level $<6.5 \%$ or $7.0 \%$ is optimally recommended, dependent on different associations [6,7].

Studies on the effects of BP variability on health outcomes have been conducted for almost a decade [8]. However, the impacts of body weight variability and the effects of starvation and body weight regain on health outcomes were first measured in starvation victims of World War II, in the so-called Minnesota Experiment [9]. The effects of glucose variability on health outcomes began to be more thoroughly and precisely evaluated after continuous glucose monitoring (CGM) systems and antidiabetic medications targeting postprandial glucose, such as dipeptidyl peptidase-4 inhibitors and glucagon-like peptide-1 receptor agonists, became available [10,11]. Studies on lipid variability were presumably initiated due to the lipid variability seen in large statin trials, and the issue of whether statin adherence affects CV outcomes $[12,13]$. In summary, most variability in CV risk factors is linked to medication adherence, the stability of the medication's effect, and the degree of lifestyle modifications.

In this study, we review the current knowledge regarding the effects of variability in CV risk factors on mortality and metabolic and CV health outcomes and discuss possible mechanisms.

\section{BODY WEIGHT VARIABILITY}

Dieting has multiple meanings, including "intentional weight loss" or "a specific eating pattern." Although obesity is consid- ered a deleterious health problem worldwide, many problems are caused by weight control measures that are too strict and the perceived need for excessive leanness. Many people with normal weight want to lose weight, and they repeat the cycle of losing and gaining weight multiple times throughout their lives. This is known as weight cycling. Recent studies have reported that although being obese has a negative impact on health, weight cycling is also dangerous for cardiometabolic health [14].

Although the exact mechanisms underlying the effects of weight cycling on health outcomes are not clear, two hypotheses address this phenomenon: the "repeated overshoot" and "increased visceral energy repartitioning" hypotheses [14,15]. Weight cycling could cause enhanced weight gain during the calorie-excess period of weight fluctuation. Sustained fluctuations in energy balance during weight cycling can lead to potential fluctuations in CV risk factors, which may increase above normal values during periods of weight regain. Therefore, fluctuations in risk factors impose an extra load on the heart and may lead to vascular injury (Fig. 1) [15]. Another plausible mechanism is repartitioning of fat and lean mass after weight cycling [16]. Weight cycling causes increased abdominal obesity and weight loss-associated adaptations in resting energy expenditure [17]. In addition, reconstitution of skeletal muscle in the trunk during spontaneous 6-month weight regain lags behind that seen in the extremities, resulting in a preferential regain of lean mass in the limbs compared with the trunk in weight-regaining subjects [18].

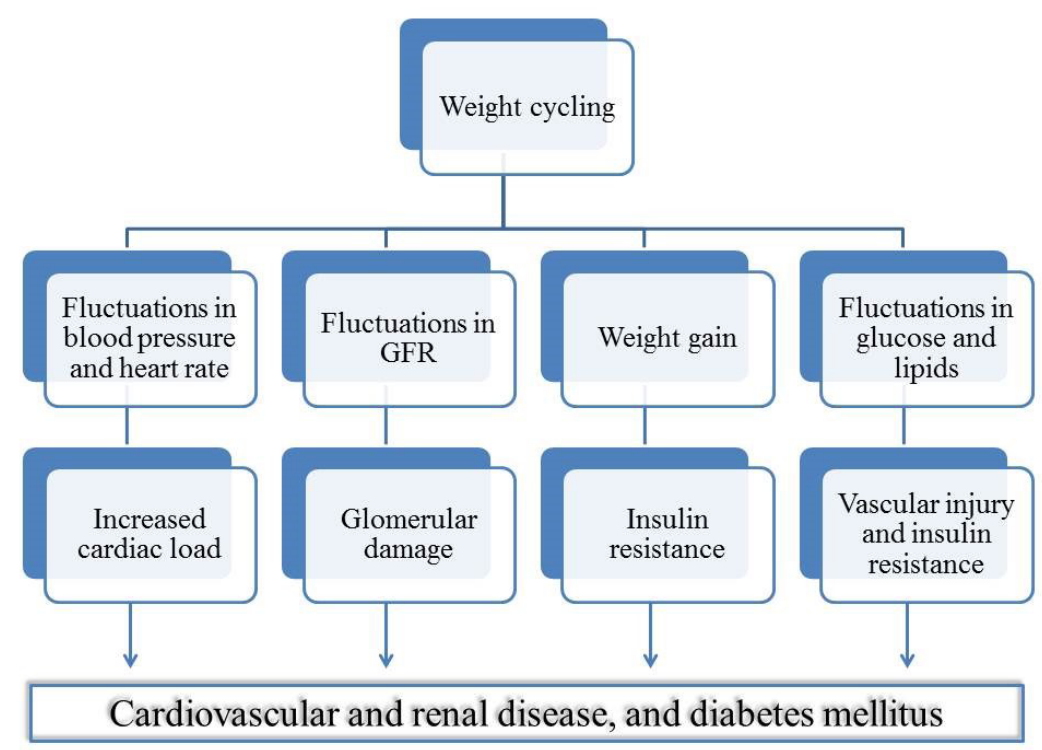

Fig. 1. Mechanisms of weight cycling effects on cardiometabolic health outcomes. Modified from Rhee [15]. GFR, glomerular filtration rate. 
The effects of weight fluctuation on mortality are controversial. In a study of 3,278 participants aged 65 years or older at baseline in United States communities in the Cardiovascular Health Study, weight cycling was associated with a significant increase in mortality [19]. In another study of 4,796 Japanese atomic bomb survivors who were followed up for 27 years, variation in body weight was associated with a 1.25 -fold increase in all-cause mortality, and this association was independent of body mass index category [20]. In a recent meta-analysis of 15 studies, the pooled overall hazard ratio (HR) for allcause mortality in the groups with the greatest weight fluctuation compared with those with most stable weight was 1.45 [21]. In contrast, in a study analyzing 55,983 men and 66,655 women in the Cancer Prevention Study II Nutrition Cohort, weight cycling was not associated with a significant increase in mortality [22]. The considerable heterogeneity among the studies explains the discrepancies between the results regarding the effects of weight fluctuation on mortality. In addition, results regarding the effects of weight on mortality must be interpreted with caution, since weight fluctuation could be caused by the presence of untreated life-threatening diseases.

Controversy also exists regarding the effects of weight fluctuation on diabetes risk. In a 6-year follow-up study in 46,634 participants from the Nurses' Health Study II, the association between weight cycling and higher rates of type 2 diabetes was no longer significant after adjustment for overall weight status [23]. In addition, in a Japanese study that analyzed weight changes over 10 years, participants who showed sustained weight gain had a 3-fold higher risk for diabetes than those whose weight remained stable [24]. However, individuals whose weight fluctuated had a lower diabetes risk than the individuals whose weight was stable. In contrast, in a study analyzing 53,088 participants of the European Prospective Investigation into Cancer and Nutrition (EPIC)-Germany cohort, in which weight cycling was defined by an annual weight change of $1.125 \mathrm{~kg}$ per year of higher, weight cycling was associated with a 1.4-fold increased risk for diabetes than stable weight [25]. In another study of 4,818 Korean health screening program participants, who were followed up for 5 years, the group with the largest annual weight change showed a 1.78-fold higher risk for diabetes than those who had the smallest weight change [26]. Individuals who were overweight or obese and had high fluctuations showed a 2.7-fold higher risk for diabetes compared with those who were lean and had low fluctuations in body weight. The result of another long-term study with a 16-year follow-up suggests that the effect of body weight fluctuation on incident diabetes depends on the presence of obesity at baseline [27]. To summarize, there is no clear consensus regarding the effect of weight fluctuation on the risk of diabetes mellitus because of the large discrepancies among the results of various studies performed with various study populations.

Weight fluctuation also affects the risk for CVD. An analysis of the Framingham Heart Study 32-year follow-up data showed that participants with highly variable body weight showed a significantly higher risk for mortality and coronary heart disease [28]. In a recent study of 9,509 participants in the Treating to New Target (TNT) trial, body weight fluctuation was significantly associated with higher mortality and a higher rate of $\mathrm{CV}$ events, independent of traditional CV risk factors, supporting previous results regarding the deleterious effects of weight fluctuation on CV event development [29].

Finally, weight fluctuation could have an influence on cancer risk. In 80,943 postmenopausal women in the Women's Health Initiative, the participants who answered that they had experienced weight cycling more than once in their life showed a higher risk for breast cancer and endometrial cancer than those who answered that they had a stable weight [30]. In contrast, in the Cancer Prevention Study II, postmenopausal women who experienced weight cycling did not show a significantly increased risk for endometrial cancer [31]. Other risk factors and surrogate markers are affected by body weight fluctuation, but they are not reviewed here (Fig. 2). More studies are needed to draw firmer conclusions.

In conclusion, weight fluctuation influences the risk of diabetes, CVD, mortality, and cancer, as observed in previous studies. However, the results are inconsistent because of differences in study populations and methods used for assessing weight cycling. The absence of a consensus on a standard definition of weight cycling or fluctuation may be the largest challenge for future studies in this field.

\section{LIPID VARIABILITY}

The benefits of lipid-lowering therapy, mainly with the use of statins, for the primary and secondary prevention of CVD have been well established by major clinical trials [32]. However, several studies have shown that discontinuation of statins increases short-term or long-term mortality and vascular events in patients with CVD [33-35]. Event rates and the risk of worse outcomes were even higher in patients who discontinued statins than in those who were not using statins. Because the rebound phenomenon induced by withdrawal of statins abrogates their 


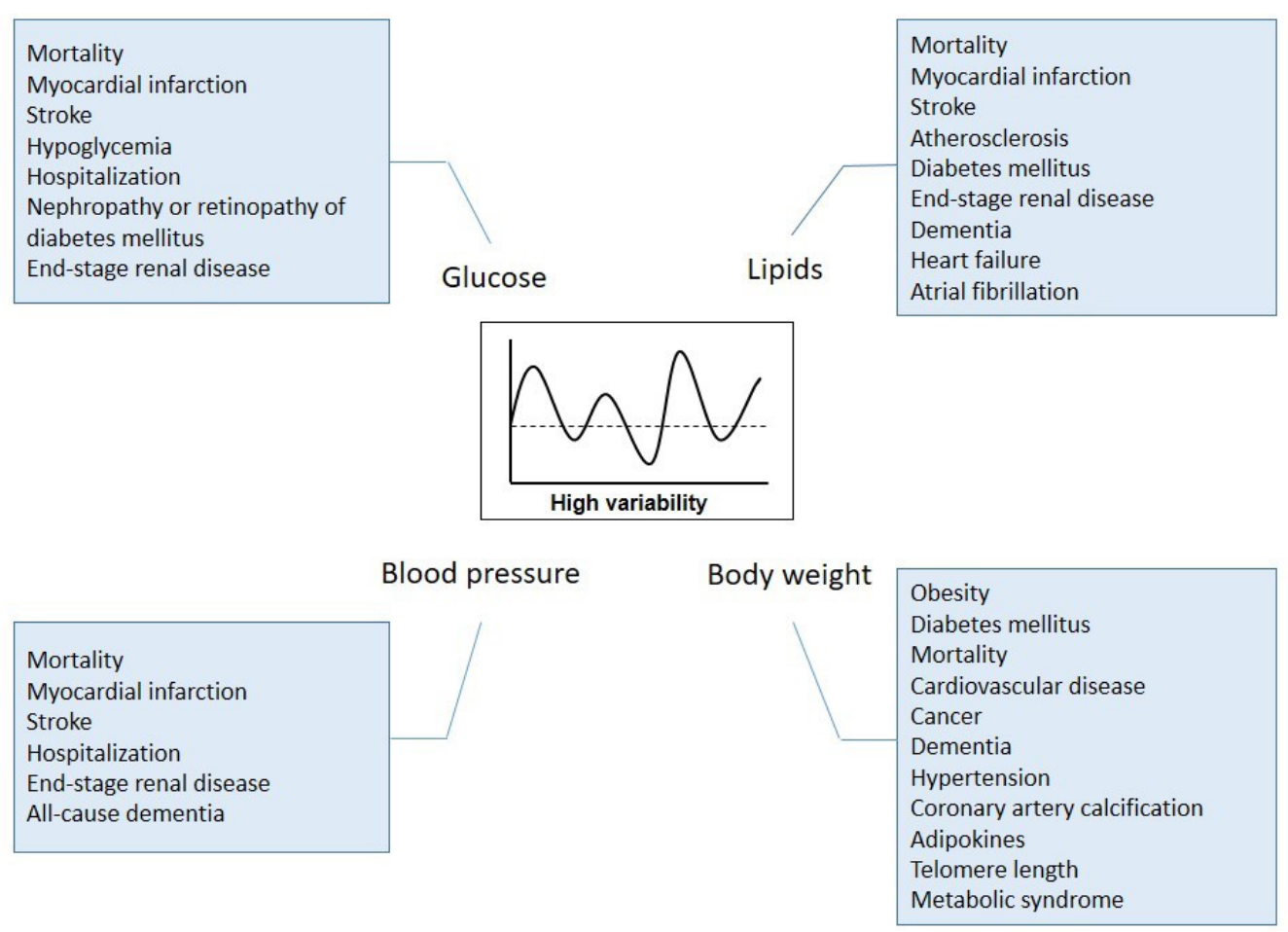

Fig. 2. High variability in body weight, lipid profile, blood pressure, and blood glucose is associated with increased risks of all-cause mortality, cardiovascular diseases, and other health outcomes.

beneficial effects on inflammation, endothelial dysfunction, hemostasis, and blood rheology in a relatively short-term period $[36,37]$, it is conceivable that unstable lipid levels might have detrimental effects.

Studies examining intra-individual day-to-day or seasonal variability in serum lipid parameters have shown that biological variability in cholesterol levels exists [38,39]. However, the clinical implications of short-term lipid variability are unknown. The clinical significance of lipid variability has emerged as a topic of interest only recently, mainly in terms of long-term (visit-to-visit) variability. The first study suggesting that total cholesterol (TC) variability should be regarded as a risk factor for mortality and CV diseases was reported in 1994 [40]. Using the Framingham Heart Study cohort, TC variability calculated from six biennial measurements was shown to be associated with all-cause mortality in men and with CVD in both sexes over a 24-year follow-up period. A post hoc analysis from the TNT trial, which enrolled patients with stable coronary artery disease, demonstrated that LDL-C variability predicted CV outcomes, independent of mean LDL-C levels [12]. A one standard deviation (SD) increase in LDL-C variability was associated with a significant increase of any coronary event by $16 \%$, any $\mathrm{CV}$ event by $11 \%$, death by $23 \%$, myocardial infarction (MI) by
$10 \%$, and stroke by $17 \%$. Subsequent reports reproduced this finding in patients who had ST-elevation MI or underwent percutaneous coronary intervention, highlighting the role of LDL$\mathrm{C}$ and high-density lipoprotein cholesterol (HDL-C) variability as an independent predictor of secondary adverse events [41, 42]. A similar phenomenon was observed in the general population without CVD. In a nationwide cohort study using a Korean claims database, the multivariable-adjusted HRs and 95\% confidence intervals (CIs) comparing the highest and lowest quartiles of TC variability were 1.26 (95\% CI, 1.24 to 1.28$)$ for allcause mortality, 1.08 (95\% CI, 1.05 to 1.11 ) for MI, and 1.11 (95\% CI, 1.08 to 1.14 ) for stroke [43]. Similarly, both low HDL-C mean levels and high HDL-C variability were associated with a higher risk for MI, stroke, and mortality, which had additive effects [44]. These associations were more prominent in people who were not taking lipid-lowering agents, suggesting that mechanisms other than medication nonadherence may explain this relationship.

More recent studies have expanded the analysis of lipid variability to include other health outcomes. Large variations in TC and HDL-C were found to be associated with a higher risk of developing diabetes $[45,46]$ or end-stage renal disease $[47,48]$. In addition, greater LDL-C variability was associated with an 
increased progression to dialysis in patients with stage 3 chronic kidney disease [49], and triglyceride variability was linked to incident microalbuminuria in patients with type 2 diabetes [50]. High lipid variability was associated with a higher risk of developing atrial fibrillation [51,52]. Analysis from the PROspective Study of Pravastatin in the Elderly at Risk (PROSPER) study using neuroimaging and cognitive function tests demonstrated that higher visit-to-visit LDL-C variability was associated with lower cognitive performance, lower cerebral blood flow, and greater white matter hyperintensity load, independent of mean LDL-C levels and statin treatment [53]. Lee et al. [54] also reported that high $\mathrm{TC}$ variability predicted all-cause dementia, Alzheimer disease, and vascular dementia in the general population without metabolic diseases.

Despite ample evidence provided by epidemiological studies, the pathophysiological mechanisms by which lipid variability leads to adverse health outcomes are still largely unknown. High cholesterol variability may induce fluctuations in the composition of vascular plaques, making them more atheromatous and vulnerable to rupture. A post hoc analysis of nine clinical trials including 4,976 patients with coronary artery disease who underwent serial intravascular ultrasonography found that greater visit-to-visit variability in LDL-C, non-HDL-C, the TC/HDL$\mathrm{C}$ ratio, and apolipoprotein $\mathrm{B}$ was significantly associated with coronary atheroma progression [55]. Endothelial dysfunction, oxidative stress, and inflammatory processes may act as mediators, as these are key pathophysiological components of many diseases induced by metabolic dysfunction. However, direct evidence is lacking and further investigation is warranted.

Polymorphisms in apolipoprotein genes have also been suggested as possible contributors to intra-individual lipid variability $[56,57]$. In a genome-wide association study, most genetic variants were not associated with lipid variability, although two suggestive signals for LDL-C variability (KIAA0391 and amiloride-sensitive cation channel 1 neuronal) and one for HDL-C variability (Dickkopf WNT signaling pathway inhibitor 3) were detected [58].

Finally, high variability in biological parameters may reflect general frailty and could be a marker of increased risk, rather than a true risk factor. However, the strong and consistent results found across many different studies support a possible causal relationship between lipid variability and adverse health outcomes.

The issue of translating these findings to clinical practice should be addressed by further investigations. Presumably, maintaining stable lifestyle patterns, including diet, exercise, and body weight, is important. It is unclear whether statin treatment or dosing affects lipid variability. In the PROSPER study, the pravastatin treatment group had higher LDL-C and HDL-C variability than the placebo group [58]. However, in the TNT trial, an atorvastatin dose of $80 \mathrm{mg} /$ day was associated with significantly lower LDL-C variability than an atorvastatin dose of $10 \mathrm{mg}$ /day dose [12]. It is worthwhile to investigate whether lipid variability induced by a monthly high-dosing schedule of PCSK9 inhibitor would cause any adverse effects $[59,60]$.

\section{BLOOD PRESSURE VARIABILITY}

$\mathrm{BP}$ is characterized by marked short-term fluctuations occurring within a 24-hour period, including beat-to-beat, hour-to-hour, and day-to-night changes. Long-term, substantial variations in BP have been shown to occur over more prolonged periods of time, for example days (day-to-day), weeks, months (visit-tovisit), seasons, and even years [61]. Previous studies on the prognostic relevance of BP variability have focused on shortterm BP variability assessed by 24-hour ambulatory BP monitoring [61]. However, evidence suggests that high day-to-day or visit-to-visit BP variability is associated with increased prevalence and severity of cardiac, vascular, and renal damage and an increased risk of fatal and nonfatal CV events [62-64]. In clinical trials, long-term BP variability is associated with $\mathrm{CV}$ outcomes to a far greater extent than short-term BP variability [61]. Long-term BP variability has been shown to be reproducible; therefore, it is not a random phenomenon [61].

Some potential mechanisms underlying long-term BP variability, particularly increased arterial stiffness, have been postulated in previous studies [65]. Factors influencing the degree of BP control, such as compliance with the prescribed therapeutic regimen and correct dosing and titration of antihypertensive treatment, or errors in BP measurement, can influence visit-tovisit $\mathrm{BP}$ variability. High variability in $\mathrm{BP}$ is a marker of the percentage of time that $\mathrm{BP}$ is not within the target range, which can contribute to a higher incidence of stroke and other CV outcomes. Among patients with hypertension, poor drug compliance is one of the most common factors affecting visit-to-visit BP variability. Visit-to-visit BP variability might be differentially affected by various classes of antihypertensive drugs [66,67]. A regimen based on the calcium-channel blocker amlodipine was found to be associated with lower intraindividual BP variability and a lower incidence of stroke than a regimen based on the beta-blocker atenolol, independent of mean BP $[66,67]$. The protective effect of antihypertensive treatment depends not only 
on the magnitude of mean BP reduction, but also on the consistency of on-treatment BP control.

However, BP variability has also been reported to be associated with the risk of CVD in individuals without hypertension $[68,69]$. BP variability is also thought to be determined by mechanisms other than drug effects or adherence. For example, higher variability in multiple biological parameters might be observed in patients with systemic conditions and generalized frailty [68]. Therefore, it is possible that high BP variability is an epiphenomenon of other systemic conditions that increase $\mathrm{CV}$ or mortality risk. Visit-to-visit variability of systolic BP exceeding 10 to $12 \mathrm{~mm} \mathrm{Hg}$ or diastolic BP variability exceeding 8 $\mathrm{mm} \mathrm{Hg}$ significantly increased the risk of hospitalization and all-cause mortality [70]. A study by Bae et al. [71] followed up a total of 8,199,089 healthy subjects for 7.9 years and showed that increased visit-to-visit BP variability was an independent risk factor for the development of end-stage renal disease.

High BP variability enhances periodic pressure loading and shear stress on the CV system and the progression of atherosclerosis [61]. Multiple adverse pathological processes including cardiac diastolic dysfunction, endothelial dysfunction, increased intima-media thickness, and arterial stiffness have been proposed as potential mechanisms to explain the association between visit-to-visit BP variability and CV outcomes [72]. In the setting of increased BP variability, increased sympathetic nerve activity plays an important role in the progression of hypertension and kidney disease. In addition, chronic sympathetic nerve hyperactivity can damage renal blood vessels by inducing smooth muscle and fibroblast proliferation in the vessel wall, reduce nitric oxide bioavailability resulting in intrarenal vasoconstriction, decrease blood flow, and worsen renal injury [71].

\section{GLUCOSE VARIABILITY}

Despite the lack of consensus regarding the most appropriate definition and tools for its assessment, glucose variability is involved in the pathogenesis of the vascular complications of diabetes [73]. There is no consensus on the gold standard for measuring glucose variability in clinical practice [73]. Two different methods of measuring intra-day glucose variability have been described in the literature: self-monitoring of blood glucose and CGM [74]. This is similar to the two methods of measuring BP variability (home BP monitoring and 24-hour ambulatory BP monitoring). Visit-to-visit fasting blood glucose (FBG) variability or visit-to-visit $\mathrm{HbA} 1 \mathrm{c}$ variability could be used as an indicator of long-term glucose variability.
In type 1 diabetes, short-term glucose variability, measured by the coefficient of variation, showed greater explanatory power for hypoglycemia than the mean glucose level when the glucose threshold was set at $54 \mathrm{mg} / \mathrm{dL}$ [75]. According to the International Consensus on Use of CGM, stable glucose levels are defined as a coefficient of variation $<36 \%$ and unstable glucose levels are defined as a coefficient of variation $\geq 36 \%$ [76]. For example, a person with a mean glucose of $150 \mathrm{mg} / \mathrm{dL}$ and a $\mathrm{SD}$ of $60 \mathrm{mg} / \mathrm{dL}$ would have a coefficient of variation of $40 \%$; this person would be categorized as having unstable glucose levels. High glucose variability is an independent risk factor for severe hypoglycemia and subsequent hospitalization in patients with diabetes [77]. The incidence and duration of hypoglycemia are associated with glucose variability [74]. In fact, hypoglycemia is associated with both macrovascular and microvascular complications in patients with type 2 diabetes mellitus [78]. $\mathrm{Pa}$ tients with type 1 diabetes are especially vulnerable to glucose variability. In type 1 diabetes, even when $\mathrm{HbA} 1 \mathrm{c}$ levels are within the target range, there is a 2.92 -fold increased risk of CV mortality [79].

Visit-to-visit variability in FBG and HbA1c was associated with the risks of microvascular and macrovascular events and all-cause mortality among patients with type 2 diabetes using data from the Action in Diabetes and Vascular Disease: Preterax and Diamicron MR Controlled Evaluation (ADVANCE) trial [80]. Variability in FBG over the course of $>7.5$ years of follow-up was a potent predictor of ischemic stroke in 28,534 patients with type 2 diabetes in the Taiwan Diabetes Study [81]. Consistency in glycemic control is important in reducing the risks of vascular events and death in type 2 diabetes.

Few previous studies have investigated the prognostic significance of glucose variability in individuals without diabetes. Recently, visit-to-visit fasting glucose variability has been reported as a risk factor for the development of type 2 diabetes [80]. We recently showed that high variability of FBG was an independent predictor of mortality and CV events among the general population [68]. For the highest quartile in FBG variability compared with the lowest quartile, the risk of all-cause mortality was $20 \%$ higher (HR, $1.20 ; 95 \%$ CI, 1.18 to 1.23 ), MI by $16 \%$ (HR, $1.16 ; 95 \% \mathrm{CI}, 1.12$ to 1.21$)$, and that of stroke was $13 \%$ higher (HR, 1.13; 95\% CI, 1.09 to 1.17). High glucose variability was associated with longer hospitalization and increased mortality in hospitalized patients, regardless of the presence of diabetes [81].

Although the exact mechanism remains to be elucidated, several mechanisms could be involved in the associations between 
visit-to-visit glycemic variability and various outcomes. Glucose fluctuation has been shown to cause overproduction of oxidative stress, an increase in inflammatory cytokines, and a loss of pancreatic beta-cells due to increased apoptotic cell death [74].

\section{CONCLUSIONS}

In this review, we examined the current literature regarding variability in four CV risk factors: body weight, lipid profiles, BP, and blood glucose. In summary, variability in most of these risk factors has generally been found to be associated with increased all-cause mortality and risk of CVD, although some discrepancies in the results exist (Fig. 2). In addition, through unknown mechanisms, fluctuations in these risk factors contribute to diabetes and obesity, even at a lower stage. Another factor that should be considered is that some of the fluctuation in risk factors is affected by adherence with the treatment regimen or the degree of lifestyle modification [82]. Most of the mechanisms suggested are based on very few consistent results, warranting further studies that might provide more definitive conclusions regarding the direct associations of variability in the abovementioned $\mathrm{CV}$ risk factors with health outcomes.

\section{CONFLICTS OF INTEREST}

No potential conflict of interest relevant to this article was reported.

\section{ORCID}

Seung-Hwan Lee https://orcid.org/0000-0002-3964-3877

Mee Kyoung Kim https://orcid.org/0000-0003-3205-9114

Eun-Jung Rhee https://orcid.org/0000-0002-6108-7758

\section{REFERENCES}

1. Grundy SM, Stone NJ, Bailey AL, Beam C, Birtcher KK, Blumenthal RS, et al. 2018 AHA/ACC/AACVPR/AAPA/ ABC/ACPM/ADA/AGS/APhA/ASPC/NLA/PCNA guideline on the management of blood cholesterol: a report of the American College of Cardiology/American Heart Association task force on clinical practice guidelines. Circulation 2019;139:e1082-143.

2. Mach F, Baigent C, Catapano AL, Koskinas KC, Casula M, Badimon L, et al. 2019 ESC/EAS guidelines for the man- agement of dyslipidaemias: lipid modification to reduce cardiovascular risk. Eur Heart J 2020;41:111-88.

3. Sabatine MS, Giugliano RP, Keech AC, Honarpour N, Wiviott SD, Murphy SA, et al. Evolocumab and clinical outcomes in patients with cardiovascular disease. N Engl J Med 2017;376:1713-22.

4. SPRINT Research Group, Wright JT Jr, Williamson JD, Whelton PK, Snyder JK, Sink KM, et al. A randomized trial of intensive versus standard blood-pressure control. N Engl J Med 2015;373:2103-16.

5. Park S. Ideal target blood pressure in hypertension. Korean Circ J 2019;49:1002-9.

6. American Diabetes Association. 6. Glycemic targets: standards of medical care in diabetes-2020. Diabetes Care 2020; 43(Suppl 1):S66-76.

7. Kim MK, Ko SH, Kim BY, Kang ES, Noh J, Kim SK, et al. 2019 Clinical practice guidelines for type 2 diabetes mellitus in Korea. Diabetes Metab J 2019;43:398-406.

8. Rothwell PM, Howard SC, Dolan E, O'Brien E, Dobson JE, Dahlof B, et al. Prognostic significance of visit-to-visit variability, maximum systolic blood pressure, and episodic hypertension. Lancet 2010;375:895-905.

9. Keys A, Brozek J, Henschel A, Mickelsen O, Taylor HL. The biology of human starvation. Minneapolis: University of Minnesota Press, 1950.

10. Chehregosha H, Khamseh ME, Malek M, Hosseinpanah F, Ismail-Beigi F. A view beyond HbA1c: role of continuous glucose monitoring. Diabetes Ther 2019;10:853-63.

11. Zenari L, Marangoni A. What are the preferred strategies for control of glycaemic variability in patients with type 2 diabetes mellitus? Diabetes Obes Metab 2013;15 Suppl 2:17-25.

12. Bangalore S, Breazna A, DeMicco DA, Wun CC, Messerli FH; TNT Steering Committee and Investigators. Visit-tovisit low-density lipoprotein cholesterol variability and risk of cardiovascular outcomes: insights from the TNT trial. J Am Coll Cardiol 2015;65:1539-48.

13. Banach M, Stulc T, Dent R, Toth PP. Statin non-adherence and residual cardiovascular risk: there is need for substantial improvement. Int J Cardiol 2016;225:184-96.

14. Montani JP, Schutz Y, Dulloo AG. Dieting and weight cycling as risk factors for cardiometabolic diseases: who is really at risk? Obes Rev 2015;16 Suppl 1:7-18.

15. Rhee EJ. Weight cycling and its cardiometabolic impact. J Obes Metab Syndr 2017;26:237-42.

16. Dulloo AG, Jacquet J. The control of partitioning between protein and fat during human starvation: its internal determi- 
nants and biological significance. Br J Nutr 1999;82:339-56.

17. Cereda E, Malavazos AE, Caccialanza R, Rondanelli M, Fatati G, Barichella M. Weight cycling is associated with body weight excess and abdominal fat accumulation: a cross-sectional study. Clin Nutr 2011;30:718-23.

18. Byrne NM, Weinsier RL, Hunter GR, Desmond R, Patterson MA, Darnell BE, et al. Influence of distribution of lean body mass on resting metabolic rate after weight loss and weight regain: comparison of responses in white and black women. Am J Clin Nutr 2003;77:1368-73.

19. Arnold AM, Newman AB, Cushman M, Ding J, Kritchevsky S. Body weight dynamics and their association with physical function and mortality in older adults: the cardiovascular health study. J Gerontol A Biol Sci Med Sci 2010;65:63-70.

20. Cologne J, Takahashi I, French B, Nanri A, Misumi M, Sadakane A, et al. Association of weight fluctuation with mortality in Japanese adults. JAMA Netw Open 2019;2:e190731.

21. Zhang Y, Hou F, Li J, Yu H, Li L, Hu S, et al. The association between weight fluctuation and all-cause mortality: a systematic review and meta-analysis. Medicine (Baltimore) 2019;98:e17513.

22. Stevens VL, Jacobs EJ, Sun J, Patel AV, McCullough ML, Teras LR, et al. Weight cycling and mortality in a large prospective US study. Am J Epidemiol 2012;175:785-92.

23. Field AE, Byers T, Hunter DJ, Laird NM, Manson JE, Williamson DF, et al. Weight cycling, weight gain, and risk of hypertension in women. Am J Epidemiol 1999;150:573-9.

24. Yokomichi H, Ohde S, Takahashi O, Mochizuki M, Takahashi A, Yoda Y, et al. Weight cycling and the subsequent onset of type 2 diabetes mellitus: 10-year cohort studies in urban and rural Japan. BMJ Open 2017;7:e014684.

25. Neamat-Allah J, Barrdahl M, Husing A, Katzke VA, Bachlechner U, Steffen A, et al. Weight cycling and the risk of type 2 diabetes in the EPIC-Germany cohort. Diabetologia 2015;58:2718-25.

26. Rhee EJ, Cho JH, Kwon H, Park SE, Park CY, Oh KW, et al. Increased risk of diabetes development in individuals with weight cycling over 4 years: the Kangbuk Samsung Health study. Diabetes Res Clin Pract 2018;139:230-8.

27. Oh TJ, Moon JH, Choi SH, Lim S, Park KS, Cho NH, et al. Body-weight fluctuation and incident diabetes mellitus, cardiovascular disease, and mortality: a 16-year prospective cohort study. J Clin Endocrinol Metab 2019;104:639-46.

28. Lissner L, Odell PM, D’Agostino RB, Stokes J 3rd, Kreger $\mathrm{BE}$, Belanger AJ, et al. Variability of body weight and health outcomes in the Framingham population. N Engl J Med 1991;
324:1839-44.

29. Bangalore S, Fayyad R, Laskey R, DeMicco DA, Messerli $\mathrm{FH}$, Waters DD. Body-weight fluctuations and outcomes in coronary disease. N Engl J Med 2017;376:1332-40.

30. Welti LM, Beavers DP, Caan BJ, Sangi-Haghpeykar H, Vitolins MZ, Beavers KM. Weight fluctuation and cancer risk in postmenopausal women: the women's health initiative. Cancer Epidemiol Biomarkers Prev 2017;26:779-86.

31. Stevens VL, Jacobs EJ, Sun J, McCullough ML, Patel AV, Gaudet MM, et al. Weight cycling and risk of endometrial cancer. Cancer Epidemiol Biomarkers Prev 2012;21:747-52.

32. LaRosa JC, Grundy SM, Waters DD, Shear C, Barter P, Fruchart JC, et al. Intensive lipid lowering with atorvastatin in patients with stable coronary disease. N Engl J Med 2005; 352:1425-35.

33. Heeschen C, Hamm CW, Laufs U, Snapinn S, Bohm M, White HD, et al. Withdrawal of statins increases event rates in patients with acute coronary syndromes. Circulation 2002; 105:1446-52.

34. Daskalopoulou SS, Delaney JA, Filion KB, Brophy JM, Mayo NE, Suissa S. Discontinuation of statin therapy following an acute myocardial infarction: a population-based study. Eur Heart J 2008;29:2083-91.

35. Colivicchi F, Bassi A, Santini M, Caltagirone C. Discontinuation of statin therapy and clinical outcome after ischemic stroke. Stroke 2007;38:2652-7.

36. Pineda A, Cubeddu LX. Statin rebound or withdrawal syndrome: does it exist? Curr Atheroscler Rep 2011;13:23-30.

37. Sposito AC, Carvalho LS, Cintra RM, Araujo AL, Ono AH, Andrade JM, et al. Rebound inflammatory response during the acute phase of myocardial infarction after simvastatin withdrawal. Atherosclerosis 2009;207:191-4.

38. Bookstein L, Gidding SS, Donovan M, Smith FA. Day-today variability of serum cholesterol, triglyceride, and highdensity lipoprotein cholesterol levels: impact on the assessment of risk according to the National Cholesterol Education Program guidelines. Arch Intern Med 1990;150:1653-7.

39. Robinson D, Bevan EA, Hinohara S, Takahashi T. Seasonal variation in serum cholesterol levels: evidence from the UK and Japan. Atherosclerosis 1992;95:15-24.

40. Kreger BE, Odell PM, D’Agostino RB, Wilson PF. Longterm intraindividual cholesterol variability: natural course and adverse impact on morbidity and mortality: the Framingham Study. Am Heart J 1994;127:1607-14.

41. Boey E, Gay GM, Poh KK, Yeo TC, Tan HC, Lee CH. Visitto-visit variability in LDL- and HDL-cholesterol is associat- 
ed with adverse events after ST-segment elevation myocardial infarction: a 5-year follow-up study. Atherosclerosis 2016;244:86-92.

42. Lee EY, Yang Y, Kim HS, Cho JH, Yoon KH, Chung WS, et al. Effect of visit-to-visit LDL-, HDL-, and non-HDL-cholesterol variability on mortality and cardiovascular outcomes after percutaneous coronary intervention. Atherosclerosis 2018;279:1-9.

43. Kim MK, Han K, Kim HS, Park YM, Kwon HS, Yoon KH, et al. Cholesterol variability and the risk of mortality, myocardial infarction, and stroke: a nationwide population-based study. Eur Heart J 2017;38:3560-6.

44. Han BH, Han K, Yoon KH, Kim MK, Lee SH. Impact of mean and variability of high-density lipoprotein-cholesterol on the risk of myocardial infarction, stroke, and mortality in the general population. J Am Heart Assoc 2020;9:e015493.

45. Rhee EJ, Han K, Ko SH, Ko KS, Lee WY. Increased risk for diabetes development in subjects with large variation in total cholesterol levels in 2,827,950 Koreans: a nationwide population-based study. PLoS One 2017;12:e0176615.

46. Lee SH, Kim HS, Park YM, Kwon HS, Yoon KH, Han K, et al. HDL-cholesterol, its variability, and the risk of diabetes: a nationwide population-based study. J Clin Endocrinol Metab 2019;104:5633-41.

47. Kim MK, Han K, Koh ES, Kim HS, Kwon HS, Park YM, et al. Variability in total cholesterol is associated with the risk of end-stage renal disease: a nationwide population-based study. Arterioscler Thromb Vasc Biol 2017;37:1963-70.

48. Koh ES, Kim M, Kim MK, Han K, Shin SJ, Kwon HS, et al. Intra-individual variability in high density lipoprotein cholesterol and risk of end-stage renal disease: a nationwide population-based study. Atherosclerosis 2019;286:135-41.

49. Lin YH, Huang JC, Wu PY, Chen SC, Chiu YW, Chang JM, et al. Greater low-density lipoprotein cholesterol variability is associated with increased progression to dialysis in patients with chronic kidney disease stage 3. Oncotarget 2017; 9:3242-53.

50. Bardini G, Innocenti M, Rotella CM, Giannini S, Mannucci E. Variability of triglyceride levels and incidence of microalbuminuria in type 2 diabetes. J Clin Lipidol 2016;10:109-15.

51. Lee HJ, Lee SR, Choi EK, Han KD, Oh S. Low lipid levels and high variability are associated with the risk of new-onset atrial fibrillation. J Am Heart Assoc 2019;8:e012771.

52. Lee SR, Choi EK, Han KD, Lee SH, Oh S. Effect of the variability of blood pressure, glucose level, total cholesterol level, and body mass index on the risk of atrial fibrillation in a healthy population. Heart Rhythm 2020;17:12-9.

53. Smit RA, Trompet S, Sabayan B, le Cessie S, van der Grond J, van Buchem MA, et al. Higher visit-to-visit low-density lipoprotein cholesterol variability is associated with lower cognitive performance, lower cerebral blood flow, and greater white matter hyperintensity load in older subjects. Circulation 2016;134:212-21.

54. Lee SH, Han K, Cho H, Park YM, Kwon HS, Kang G, et al. Variability in metabolic parameters and risk of dementia: a nationwide population-based study. Alzheimers Res Ther 2018;10:110.

55. Clark D 3rd, Nicholls SJ, St John J, Elshazly MB, Kapadia SR, Tuzcu EM, et al. Visit-to-visit cholesterol variability correlates with coronary atheroma progression and clinical outcomes. Eur Heart J 2018;39:2551-8.

56. Porkka KV, Taimela S, Kontula K, Lehtimaki T, Aalto-Setala K, Akerblom HK, et al. Variability gene effects of DNA polymorphisms at the apo B, apo A I/C III and apo E loci on serum lipids: the Cardiovascular Risk in Young Finns Study. Clin Genet 1994;45:113-21.

57. Pereira MA, Weggemans RM, Jacobs DR Jr, Hannan PJ, Zock PL, Ordovas JM, et al. Within-person variation in serum lipids: implications for clinical trials. Int J Epidemiol 2004;33:534-41.

58. Smit RAJ, Jukema JW, Postmus I, Ford I, Slagboom PE, Heijmans BT, et al. Visit-to-visit lipid variability: clinical significance, effects of lipid-lowering treatment, and (pharmaco) genetics. J Clin Lipidol 2018;12:266-76.

59. Ballantyne CM, Neutel J, Cropp A, Duggan W, Wang EQ, Plowchalk D, et al. Results of bococizumab, a monoclonal antibody against proprotein convertase subtilisin/kexin type 9, from a randomized, placebo-controlled, dose-ranging study in statin-treated subjects with hypercholesterolemia. Am J Cardiol 2015;115:1212-21.

60. Kastelein JJ, Nissen SE, Rader DJ, Hovingh GK, Wang MD, Shen T, et al. Safety and efficacy of LY3015014, a monoclonal antibody to proprotein convertase subtilisin/ kexin type 9 (PCSK9): a randomized, placebo-controlled phase 2 study. Eur Heart J 2016;37:1360-9.

61. Parati G, Ochoa JE, Lombardi C, Bilo G. Assessment and management of blood-pressure variability. Nat Rev Cardiol 2013;10:143-55.

62. Gosmanova EO, Mikkelsen MK, Molnar MZ, Lu JL, Yessayan LT, Kalantar-Zadeh K, et al. Association of systolic blood pressure variability with mortality, coronary heart disease, stroke, and renal disease. J Am Coll Cardiol 2016;68: 
1375-86.

63. Muntner P, Whittle J, Lynch AI, Colantonio LD, Simpson LM, Einhorn PT, et al. Visit-to-visit variability of blood pressure and coronary heart disease, stroke, heart failure, and mortality: a cohort study. Ann Intern Med 2015;163: 329-38.

64. Choi S, Shin J, Choi SY, Sung KC, Ihm SH, Kim KI, et al. Impact of visit-to-visit variability in systolic blood pressure on cardiovascular outcomes in Korean National Health Insurance Service-National Sample Cohort. Am J Hypertens 2017;30:577-86.

65. Nagai M, Hoshide S, Ishikawa J, Shimada K, Kario K. Visitto-visit blood pressure variations: new independent determinants for carotid artery measures in the elderly at high risk of cardiovascular disease. J Am Soc Hypertens 2011;5:18492.

66. Webb AJ, Fischer U, Mehta Z, Rothwell PM. Effects of antihypertensive-drug class on interindividual variation in blood pressure and risk of stroke: a systematic review and meta-analysis. Lancet 2010;375:906-15.

67. Rothwell PM, Howard SC, Dolan E, O’Brien E, Dobson JE, Dahlof B, et al. Effects of beta blockers and calcium-channel blockers on within-individual variability in blood pressure and risk of stroke. Lancet Neurol 2010;9:469-80.

68. Kim MK, Han K, Park YM, Kwon HS, Kang G, Yoon KH, et al. Associations of variability in blood pressure, glucose and cholesterol concentrations, and body mass index with mortality and cardiovascular outcomes in the general population. Circulation 2018;138:2627-37.

69. Kikuya M, Ohkubo T, Metoki H, Asayama K, Hara A, Obara $\mathrm{T}$, et al. Day-by-day variability of blood pressure and heart rate at home as a novel predictor of prognosis: the Ohasama study. Hypertension 2008;52:1045-50.

70. Basson MD, Klug MG, Hostetter JE, Wynne J. Visit-to-visit variability of blood pressure is associated with hospitalization and mortality in an unselected adult population. Am J Hypertens 2018;31:1113-9.

71. Bae EH, Lim SY, Han KD, Oh TR, Choi HS, Kim CS, et al. Association between systolic and diastolic blood pressure variability and the risk of end-stage renal disease. Hypertension 2019;74:880-7.

72. Seo SM, Chung WB, Choi IJ, Koh YS, Ihm SH, Kim PJ, et al. Visit-to-visit variability of systolic blood pressure pre- dicts all-cause mortality in patients received percutaneous coronary intervention with drug-eluting stents. Heart Vessels 2018;33:489-97.

73. Nusca A, Tuccinardi D, Albano M, Cavallaro C, Ricottini E, Manfrini S, et al. Glycemic variability in the development of cardiovascular complications in diabetes. Diabetes Metab Res Rev 2018;34:e3047.

74. Suh S, Kim JH. Glycemic variability: how do we measure it and why is it important? Diabetes Metab J 2015;39:273-82.

75. Monnier L, Wojtusciszyn A, Molinari N, Colette C, Renard E, Owens D. Respective contributions of glycemic variability and mean daily glucose as predictors of hypoglycemia in type 1 diabetes: are they equivalent? Diabetes Care 2020;43: 821-7.

76. Battelino T, Danne T, Bergenstal RM, Amiel SA, Beck R, Biester T, et al. Clinical targets for continuous glucose monitoring data interpretation: recommendations from the international consensus on time in range. Diabetes Care 2019;42: 1593-603.

77. Peled S, Pollack R, Elishoov O, Haze A, Cahn A. Association of inpatient glucose measurements with amputations in patients hospitalized with acute diabetic foot. J Clin Endocrinol Metab 2019;104:5445-52.

78. Hirakawa Y, Arima H, Zoungas S, Ninomiya T, Cooper M, Hamet $\mathrm{P}$, et al. Impact of visit-to-visit glycemic variability on the risks of macrovascular and microvascular events and all-cause mortality in type 2 diabetes: the ADVANCE trial. Diabetes Care 2014;37:2359-65.

79. Lin CC, Yang CP, Li CI, Liu CS, Chen CC, Lin WY, et al. Visit-to-visit variability of fasting plasma glucose as predictor of ischemic stroke: competing risk analysis in a national cohort of Taiwan Diabetes Study. BMC Med 2014;12:165.

80. Kim JA, Lee JS, Chung HS, Roh E, Lee YB, Hong SH, et al. Impact of visit-to-visit fasting plasma glucose variability on the development of type 2 diabetes: a nationwide population-based cohort study. Diabetes Care 2018;41:2610-6.

81. Akirov A, Diker-Cohen T, Masri-Iraqi H, Shimon I. High glucose variability increases mortality risk in hospitalized patients. J Clin Endocrinol Metab 2017;102:2230-41.

82. Gupta R, Wood DA. Primary prevention of ischaemic heart disease: populations, individuals, and health professionals. Lancet 2019;394:685-96. 